\title{
Günstigere Prognose unter Antipsychotika der zweiten Generation
}

Fragestellung: Im Rahmen der Studie wurden das klinische Profil sowie die Mortalität des malignen neuroleptischen Syndroms (MNS) unter klassischen Neuroleptika (erste Generation) und atypischen Antipsychotika (zweite Generation) verglichen.

Hintergrund: Das MNS ist eine seltene Nebenwirkung von Antipsychotika. Obwohl sowohl die Inzidenz als auch die Mortalität seit Einführung der zweiten Generation oder atypischen Antipsychotika scheinbar abgenommen hat, bleibt das rasche Erkennen dieser lebensbedrohlichen Komplikation für die Prognose von höchster Priorität. Klassischerweise ist das MNS durch vegetative Symptome in Form von Hyperthermie und Tachykardie, extrapyramidal-motorischen Störungen mit ausgeprägtem Rigor sowie Bewusstseinsstörungen wie Verwirrtheit und Stupor bis hin zum Koma gekennzeichnet. Es kommt zu Serumkreatininkinase- und Transaminasenanstieg, Elektrolytentgleisungen, Myoglobinurie bis zur Rhabdomyolyse mit konsekutiver renaler Stö-

Trollor JN, Chen X, Chitty K, Sachdev PS. Comparison of neuroleptic malignant syndrome induced by first- and second-generation antipsychotics. Br J Psychiatry 2012; 201: 52-6 rung. Fallberichte der letzten Jahre geben Hinweise darauf, dass sich das MNS unter Antipsychotika der zweiten Generation in atypischer Weise klinisch präsentiert. So scheinen Clozapin-indu- zierte MNS mit weniger ausgeprägtem Rigor und geringerer Kreatinkinaseerhöhung einherzugehen, während Risperidoninduzierte MNS seltener Hyperthermie aufzuweisen scheinen.

Patienten und Methodik: Mithilfe der australischen Adverse Drug Reaction Advisory Committee Database (ADRAC) wurden 208 Patienten identifiziert, bei denen von April 1994 bis September 2010 unter Monotherapie mit klassischen Neuroleptika oder atypischen Antipsychotika MNS aufgetreten waren. Verglichen wurden die klinischen Charakteristika der MNS sowie die Mortalität.

Ergebnisse: Die Patienten mit MNS unter Atypika waren jünger, hatten häufiger eine psychotische Störung und zeigten eine geringere Ausprägung von Rigor und anderen extrapyramidalmotorischen Symptomen. Dieser Unterschied war in erster Linie durch die Clozapin-induzierten MNS begründet. Die Mortalität war deutlich geringer unter atypischen Antipsychotika (3\%) versus traditionellen Neuroleptika (16,3\%).

Schlussfolgerungen: Das MNS unter Atypika ist weitestgehend vergleichbar mit dem unter traditionellen Neuroleptika, wobei die seltenen Clozapin-induzierten MNS geringere extrapyramidal-motorische Symptome zeigen. Die Mortalität ist unter Antipsychotika der zweiten Generation deutlich geringer.

\section{- Kommentar von Prof. Dieter F. Braus}

\section{MNS treten auch unter atypischen Antipsychotika auf}

Diese Studie analysiert die derzeit größte Fallserie von malignen neuroleptischen Syndromen unter atypischen Antipsychotika anhand einer gut etablierten Datenbank (Adverse Drug Reaction Advisory Committee Database (ADRAC). Die Ergebnisse stimmen mit einer kleineren, vergleichbaren Untersuchung einer japanischen Datenbank überein [1] und verdeutlichen, dass insbesondere die extrapyramidal-motorischen Symptome bei dieser schwerwiegenden psychiatrischen Komplikation unter atypischen Antipsychotika verglichen mit klassischen Neuroleptika in den Hintergrund treten, bei insgesamt geringerer Mortalität. Die größte Limitierung dieser Untersuchung ist die mangelnde Standardisierung der Diagnosen und therapeutischen Interventionen sowie Unvollständigkeit relevanter klinischer Daten in der Datenbank. Außerdem lassen sich aufgrund der geringen Fallzahl keine Aussagen zur klinischen Präsentation von MNS unter Amisulprid, Aripiprazol, Ziprasidon und Paliperidon erheben. Die Studie mahnt jedoch alle Kliniker, sich daran zu erinnern, dass MNS auch unter Antipsychotika der zweiten Generation auftreten, und dass bei klinischem Verdacht die extrapyramidal-motorischen Störungen, insbesondere der klassische Rigor, weniger prominent sind. Die Studie gibt leider keine Hinweise darauf, den Pathomechanismus, der mit Dopamin, Acetylcholin und der zentralen Thermoregulation zu tun hat, besser zu verstehen [2].
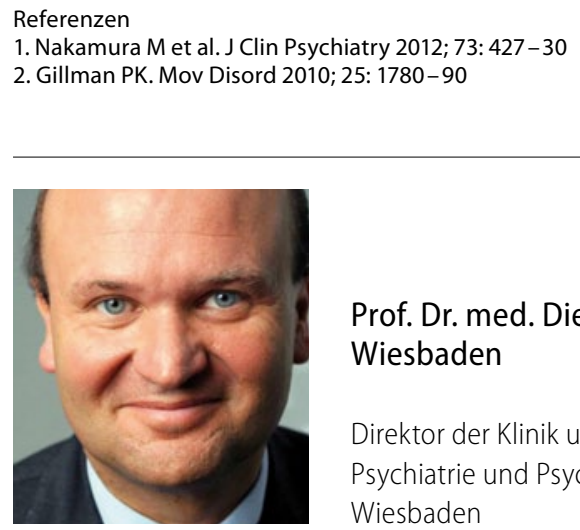

Prof. Dr. med. Dieter F. Braus, Wiesbaden

Direktor der Klinik und Poliklinik für Psychiatrie und Psychotherapie, Wiesbaden 